\title{
Smart (In-home) Power Scheduling for Demand Response on the Smart Grid
}

\author{
Gang Xiong, Chen Chen, Shalinee Kishore, Member, IEEE, and Aylin Yener, Member, IEEE
}

\begin{abstract}
This paper proposes a power scheduling-based communication protocol for in-home appliances connected over home area network and receiving real-time electricity prices via a smart meter. Specifically, a joint media access and appliance scheduling approach is developed to allow appliances to coordinate power usage so that total demand for the home is kept below a target value. Two types of appliances are considered: 1) "real-time" which consume power as they desire; and 2) "schedulable" which can be turned on at a later time. Simulation results indicate that for an appropriate target total power consumption, our scheme leads to a reduced peak demand for the home and produces a demand that is more level over time.
\end{abstract}

Index Terms-Smart grid, power management, demand response.

\section{INTRODUCTION}

$\mathbf{T}$ HE emerging Smart Grid will provide residential users flexibility in controlling their electricity costs. A primary driving force is the smart meter, which can deliver "real-time" electricity prices to homes, potentially every fifteen minutes [1]. The customer can make use of this information via an inhome energy management controller (EMC), which uses both prices and user preferences to control power usage across the home. The EMC may be standalone or embedded either in the smart meter or in appliances. At the same time, customers may participate in Direct Load Control (DLC) that allows utilities to control some power consumption within a home during peak usage (thereby bypassing or even replacing the EMC) as a part of an energy savings subscription plan.

Such methods for controlling electricity consumption are part of demand response, which relies on varying price of electricity to reduce peak demand. Reduced peak demand lowers electricity bills and benefits utilities by reducing complexity of grid stability, occurrences of equipment failures, brownouts, and blackouts. It also enables utilities to comply with government mandates to cut peak demand, e.g., [2].

In this paper, we consider mechanisms to reduce demand peaks within a home comprised of smart appliances that communicate with one another over a home-area network (HAN). Specifically, we exploit the fact that many home appliances offer some degree of flexibility regarding when they are operated. For example, homeowners may be indifferent as to when the dishwasher or clothes dryer run, as long

Gang Xiong, Chen Chen and Shalinee Kishore are with the Department of Electrical and Computer Engineering, Lehigh University, Bethlehem, PA 18015 USA. E-mail:\{gxiong, cchen, skishore\}@1ehigh.edu.

Aylin Yener is with the Department of Electrical Engineering, The Pennsylvania State University, University Park, PA 16802 USA. Email: yener@ee.psu.edu.

Manuscript received xx, 2010; revised xx, 201x.

978-1-61284-220-2/11/\$26.00 @2011 IEEE as the cycle is complete by a prescribed time. We use this flexibility to adapt total power consumption based on current electricity prices. That is, we assume real-time prices are used to calculate, $\mathcal{P}_{\max , t}$, a target power consumption level for the home at time $t$. For example, this target value can be obtained by incorporating current electricity prices with the customer's budgetary preference (e.g., no more than $\$ x$ to be spent on electricity bills this month) and their usage pattern (e.g., total cost incurred thus far this month and expected usage for the remainder). Or it could be a power level set by the utility, e.g., as a part of a DLC program. Our joint media access and appliance scheduling approach gives an access method for the common control channel of the HAN so that appliances can coordinate usage and meet the target $\mathcal{P}_{\max , t}$. All appliances that are currently operating are termed "active" and a leader node (e.g., the EMC, one of the active appliances or the utility) 1) monitors power consumption via load control messages sent on the control channel; and 2) admits new appliances to this active set. Two types of appliances are accommodated. First are "real-time" which are granted immediate access to the active set (i.e, those that must be turned on immediately); the second are "schedulable" which can be turned on at a later time. Results show that for an appropriate $\mathcal{P}_{\max , t}$, our scheme leads to a reduced peak demand for the home and produces a demand that is more level over time.

Unlike existing HAN media access protocols (e.g., Wi-Fi, G.hn, Zigbee) which can accommodate several communication applications, our proposed scheme is tailored for the load control problem. Given the large time-scale for pricing updates, relatively short power control messages are only occasionally exchanged in this scheme. Thus, the approach presented here can also be implemented on (high rate) HAN's that may support other applications (e.g., sharing Internet access) as long as load control messages are given transmission priority. And unlike existing studies on EMC design (e.g., [3][4][5]), the energy management approach presented includes a joint design of the underlying communication algorithm. Further, our scheme can be implemented over any existing HAN with a common control channel. This is unlike existing EMC studies that focus on power-line based HANs, e.g., [6][7].

The remainder of the paper is organized as follows: Section II describes our proposed power scheduling protocol. Section III discusses the power scheduling with real-time appliances. Simulation results are presented in Section IV and we conclude our discussion in Section V. 


\section{Power Scheduling Protocol for Demand RESPONSE IN SMART GRID SYSTEM}

In the model studied here, we assume that a desired $\mathcal{P}_{\max , t}$ has been determined for the residence. As noted above, this can be calculated using current electricity prices (which in turn depends on utility's current power supply) and the customer's budgetary and usage preferences. In Section II.C we present an approach for setting $\mathcal{P}_{\max , t}$. The goal of the scheme presented here is to exploit the "schedulability" of smart appliances to bring peak usage at or below the threshold power level. We note, however, that certain usage patterns will not permit total usage below $\mathcal{P}_{\text {max }, t}$; in such cases, our scheme will attempt to reduce overall usage close to $\mathcal{P}_{\max , t}$ with the understanding that the customer will have to pay more for the higher demand.

\section{A. Protocol Description}

We assume a HAN with $N$ appliances that share a common control channel. Time is segmented into scheduling frames, which are on the order of several $(\sim 5)$ minutes long and there may be multiple scheduling frames in the duration between pricing updates. Given the long frame duration and short packet duration in this proposed scheme, we can assume that transmission collisions occur on the control channel with negligible probability.

We define the active set $\left(\mathcal{A}_{t}\right)$ as the set of appliances which are operating during time frame $t$. We also assign a leader appliance $\left(\ell_{t}\right)$ that broadcasts a beacon signal and makes admission control decisions during frame $t$. This leader can be the EMC or the utility sending DLC messages to homes during peak hours. In the absence of a central EMC, the leader can be one of the appliances in $\mathcal{A}_{t}$; later in this section, we present a possible leader assignment approach.

We consider two types of appliances in the home. The first are real-time appliances that must join the active set immediately. These can be "critical" devices (e.g., medical) or devices that the customer decides must be used immediately (e.g., a laptop). The second are schedulable appliances that can be started with a delay ${ }^{1},{ }^{2}$. To ease discussion, we first assume only schedulable appliances and then in the following section, we will discuss modifications to include real-time appliances.

Fig. 1 shows the frame structure of proposed media access and scheduling protocol. The frame consists of three phases: power update, power request and power scheduling, each lasting $T_{1}, T_{2}$ and $T_{3}$ seconds, respectively.

In the power update phase, any active appliance which finishes its power usage sends a power update message (PUM) packet, which indicates the amount of power the appliance is releasing. Any appliance that completes operation during the other two phases, waits till the next power update phase to send out this message. At this end of the power update phase,

\footnotetext{
${ }^{1}$ For simplicity, we assume no delay constraints in the admission control schemes studied below.

${ }^{2}$ In practice, there is another category of home appliances: Those that cycle on and off. In Section II.C, we discuss how such appliances can be accommodated by our proposed scheme by modifying the calculation of $\mathcal{P}_{\max , t}$.
}

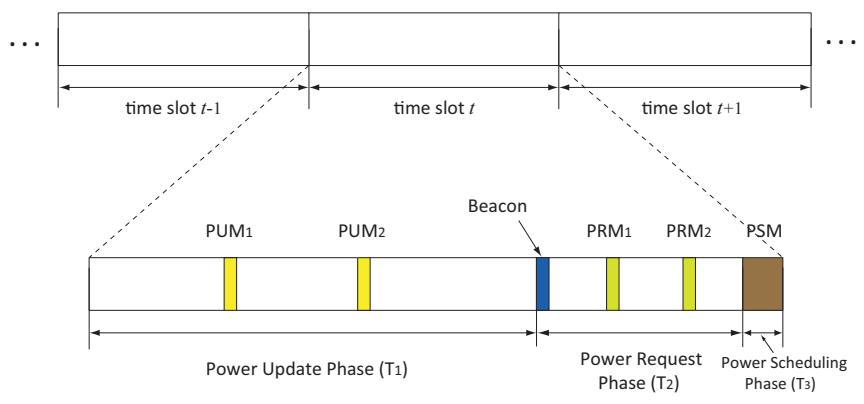

Fig. 1. Power scheduling protocol in smart grid system.

the leader calculates the remaining power budget as

$$
\mathcal{P}_{r}^{(t)}=\mathcal{P}_{\text {max }, t}-\sum_{j \in \mathcal{A}_{t}} \mathcal{P}_{j}
$$

and broadcasts this information in the beacon signal, which marks the start of the second phase. Here, $\mathcal{P}_{j}$ is the power usage for appliance $j$ which just completed its power use. $\mathcal{P}_{\text {max }, t}$ is the total power budget for frame $t$. Details of the PUM packet are given in Fig. 2 and Table I.

\begin{tabular}{|l|l|l|l|l|}
\hline ID & Mes_Ind & P_Lev & P_Dur & Res \\
\hline
\end{tabular}

Fig. 2. Power update message format.

TABLE I

POWER UPDATE MESSAGE ELEMENT

\begin{tabular}{l|l}
\hline ID & Appliance ID \\
\hline Mes_Ind & $\begin{array}{r}\text { Message indicator, 0: power update; 1: power request; } \\
\text { 2: power scheduling; 3: power curtailment. }\end{array}$ \\
\hline P_Lev & Power usage level \\
\hline P_Dur & Power usage duration \\
\hline Res & Reserved bit \\
\hline
\end{tabular}

The power request phase is divided into time slots. In the first slot, the leader sends out its beacon signal indicating the remaining power budget. Any (schedulable) appliance which wishes to join the active set listens to the control channel and, upon receiving the leader's beacon signal, compares its requested power level with the remaining power budget. If its requested demand exceeds remaining power budget, i.e., $\mathcal{P}_{i} \geq \mathcal{P}_{r}^{(t)}$, then appliance $i$ will defer requesting access to the active set until next time frame. Otherwise, this appliance attempts joining the active set in the current frame. To do so, it waits for a random number of time slots (uniformly distributed in $[1, w]$, where $w$ is the number of slots $\mathbf{n}$ this phase) and then sends out a power request message (PRM) packet to request the power usage. During this time, leader monitors the control channel and collects all the power request information. Details of PRM packet are shown in Fig. 3 and Table II.

In the power scheduling phase, the leader appliance makes an admission control decision, i.e., decides which (schedulable) appliances can join the active set. Various admission control methods (ACM) will be discussed later in this section. The 


\begin{tabular}{|l|l|l|l|l|l|l|l|}
\hline ID & Mes_Ind & Pr_Ind & P_Lev & P_Dur & P_Deadline & Num_Try & Res \\
\hline
\end{tabular}

Fig. 3. Power request message format.

TABLE II

POWER REQUEST MESSAGE ELEMENT

\begin{tabular}{l|l}
\hline Pr_Ind & $\begin{array}{r}\text { Priority indicator, 0: schedulable appliance; } \\
\text { 1: real-time appliance. }\end{array}$ \\
\hline P_Deadline & Power usage deadline \\
\hline Num_Try & Number of request reattempts \\
\hline
\end{tabular}

leader appliance then broadcasts a power scheduling message (PSM) packet. All appliances including active appliances listen to this PSM packet. The newly admitted appliances (in the PSM) start consuming power immediately. Any non-admitted appliances will wait until next frame to request again. Details of PSM packet are shown in Fig. 4 and Table III.

\begin{tabular}{|l|l|l|l|l|l|l|l|l|}
\hline ID & Mes_Ind & Num_Act & A_ID1 & $\cdots$ & A_IDM & P_T_Lev & Lead_ID & Res \\
\hline
\end{tabular}

Fig. 4. Power scheduling message format.

TABLE III

POWER SCHEDULING MESSAGE ELEMENT

\begin{tabular}{l|l}
\hline Num_Act & Number of new active appliances \\
\hline A_ID & New active appliance ID \\
\hline P_T_Lev & Total power usage level \\
\hline Lead_ID & Leader appliance in the next time slot \\
\hline
\end{tabular}

In the case that neither the EMC nor the utility is the leader, the role of the leader appliance must be established. In this case, we can assume that initially the first appliance which powers on will be the leader and sends out the beacon signal to synchronize all appliances in the system. Subsequently, the last admitted appliance (the one listed last in the PSM message) is the leader for the upcoming frame. Thus, the role of the leader could potentially change from one frame to the next.

\section{B. Admission Control Methods}

Let us define the following appliance sets:

- Feasible appliance set $\left(\mathcal{F}_{t}\right)$ : set of appliances whose individual requested power level is less than remaining power budget during time frame $t$;

- Admissible appliance set $\left(\mathcal{D}_{t}\right)$ : set of appliances in $\mathcal{F}_{t}$ whose PRM packets are received correctly by the leader during time frame $t$;

- New admitted appliance set $\left(\mathcal{N}_{t}\right)$ : set of appliances in $\mathcal{D}_{t}$ can be granted admission by leader during time frame $t$.

It is straightforward to see that when the total request power is not greater than the remaining power budget, i.e.,

$$
\sum_{i \in \mathcal{D}_{t}} \mathcal{P}_{i} \leq \mathcal{P}_{\max , t}-\sum_{j \in \mathcal{A}_{t}} \mathcal{P}_{j}
$$

the leader appliance can admit all appliances in the admissible set, $\mathcal{D}_{t}$. When the total request power is greater than the remaining budget, however, we consider the following ACMs:

1) Random ACM: Here, the leader randomly removes appliances from the admissible set, $\mathcal{D}_{t}$ to meet $\mathcal{P}_{\max , t}$ :

(a): Randomly remove appliance $i$ from $\mathcal{D}_{t}$. Let $\mathcal{D}_{t}=$ $\mathcal{D}_{t} \backslash\{i\}$ and calculate

$$
\mathcal{P}_{l}=\sum_{i \in \mathcal{D}_{t} \cup \mathcal{A}_{t}} \mathcal{P}_{i}-\mathcal{P}_{\text {max }, t}
$$

(b): If $\mathcal{P}_{l} \leq 0$, set $\mathcal{N}_{t}=\mathcal{D}_{t}$ and stop; else go to (a).

Note when $\mathcal{D}_{t} \neq \emptyset$, then $\mathcal{N}_{t} \neq \emptyset$ since all members of $\mathcal{D}_{t}$ conducted individual power check in the power request phase.

2) Max-Power Oriented ACM: In this case, the leader appliance maximizes the total additional power supported without exceeding the remaining power budget:

$$
\begin{array}{ll}
\max & \sum_{i \in \mathcal{D}_{t}} x_{i} \mathcal{P}_{i} \\
\text { s.t. } & \sum_{i \in \mathcal{D}_{t}} x_{i} \mathcal{P}_{i} \leq \mathcal{P}_{\text {max }, t}-\sum_{j \in \mathcal{A}_{t}} \mathcal{P}_{j} \\
& x_{i} \in\{0,1\} .
\end{array}
$$

This is a traditional 0-1 knapsack problem and can be solved using various existing approaches, e.g., [8]. The new admitted set can be chosen as $\mathcal{N}_{t}=\left\{i \mid x_{i}=1, i \in \mathcal{D}_{t}\right\}$.

3) Max-Appliance Oriented ACM: Here, the leader selects appliances so that a maximum number of them are supported:

$$
\begin{array}{ll}
\max & \sum_{i \in \mathcal{D}_{t}} x_{i} \\
\text { s.t. } & \sum_{i \in \mathcal{D}_{t}} x_{i} \mathcal{P}_{i} \leq \mathcal{P}_{\text {max }, t}-\sum_{j \in \mathcal{A}_{t}} \mathcal{P}_{j} \\
& x_{i} \in\{0,1\} .
\end{array}
$$

We see that this optimization problem can be solved by first sorting the individual requested power from the lowest to the highest. Without loss of generality, we assume that $\mathcal{P}_{1} \leq \mathcal{P}_{2} \leq$ $\cdots \leq \mathcal{P}_{\left|\mathcal{D}_{t}\right|}$, where $|\cdot|$ denotes the cardinality of a set. Then,

$$
\mathcal{N}_{t}=\left\{\left\{1, \cdots, \max \left\{i_{\mathcal{S}}\right\}\right\} \mid \sum_{k=1}^{i_{\mathcal{S}}} \mathcal{P}_{k} \leq \mathcal{P}_{\max , t}-\sum_{j \in \mathcal{A}_{t}} \mathcal{P}_{j}\right\} .
$$

\section{Determination of $\mathcal{P}_{\max , t}$}

Here we present an approach to compute the total power budget $\mathcal{P}_{\text {max }, t}$ for time period $t$. We formulate an optimization problem which aims to minimize the total cost of electricity on a daily scale by adjusting the total power budget for time $t$. Without loss of generality, we consider the time period $t$ to be one hour long. We denote the price of electricity (set by the utility) as $\kappa_{t} \$ / \mathrm{kWh}$ for hour $t$. The power budget $\mathcal{P}_{\text {max }, t}$ can then be found as the solution to the following:

$$
\begin{array}{ll}
\min & \sum_{t=1}^{24} \kappa_{t} \mathcal{P}_{\text {max }, t} \\
\text { s.t. } & \mathcal{L}_{t} \leq \mathcal{P}_{\text {max }, t} \leq \mathcal{M}_{t}, t=1, \cdots, 24, \\
& \sum_{t=1}^{24} \mathcal{P}_{\text {max }, t} \geq \mathcal{E}_{0} .
\end{array}
$$


where $\mathcal{E}_{0}$ is the minimum total power consumption for one day; $\mathcal{L}_{t}$ and $\mathcal{M}_{t}$ denote the minimum and maximum power consumption level at time $t$, respectively. These three parameters can be determined, for example, by the EMC using learning algorithms that follow the consumer's usage patterns. Alternatively, we may select $\mathcal{L}_{t}$ as the aggregate power consumption level by real-time appliances, i.e.,

$$
\mathcal{L}_{t}=\sum_{i \in \mathcal{R}_{t}} \mathcal{P}_{i}+\delta_{t},
$$

where $\mathcal{R}_{t}$ is the set of real-time appliances at time $t$ and $\delta_{t}$ is the slack power level that corresponds to the real-time appliances with cycle power (e.g. air conditioners). Such a selection of $\mathcal{L}_{t}$ guarantees there always sufficient power budget to accommodate the real-time appliances. Additionally, $\mathcal{M}_{t}$ may be provided by the utility to avoid aggregate peak power at the power grid.

\section{Power Scheduling Protocol with Real-Time APPLIANCES}

We now consider modifications to our scheme so as to support real-time appliances. We assume that a real-time appliance will start operating immediately and will send out a PRM message (with the priority bit set 1 , indicating its realtime status) over the control channel. This message can be sent out at any time. If when the message is sent out there is sufficient remaining power to support the real-time appliance, then the proposed algorithm and the leader's admission control are not deeply affected. The leader simply has to adjust the remaining power level (to include the real-time device) and use this adjusted level for admission control. Since all active appliances are listening to the control channel, they too can adjust their measurement of remaining power to account for the real-time appliance's power usage.

On the other hand, if there is not enough remaining power to accommodate the current usage, then the leader must employ a curtailment control method (CCM). That is, the leader must construct a curtailable appliance set, $\mathcal{C}_{t}$ from the active set for each time frame $t$. To do so, it will have to accommodate for the characteristics and power cycle of each appliance individually (e.g., a dishwasher in the middle of its wash cycle may not be curtailed but it may in the middle of its heated dry cycle, etc.). If $\mathcal{C}_{t} \neq \emptyset$, then the leader must select which member of $\mathcal{C}_{t}$ must be removed from $\mathcal{A}_{t}$. The leader broadcasts such curtailment messages using the power curtailment message (PCM) format shown in Fig. 5 and Table IV.

\begin{tabular}{|l|l|l|l|l|l|l|l|}
\hline ID & Mes_Ind & Num_Cur & C_ID1 & $\cdots$ & C_IIDM & P_T_Lev & Res \\
\hline
\end{tabular}

Fig. 5. Power curtailment message format.

To determine which active appliances in the set $\mathcal{C}_{t}$ should be curtailed, the leader may decide based on various criterion. For example,

1) Under random curtailment, the leader can remove appliances randomly from $\mathcal{C}_{t}$.
TABLE IV

POWER CURTAILMENT MESSAGE ELEMENT

\begin{tabular}{l|l}
\hline Num_Cur & Number of curtailment appliances \\
\hline C_ID & Curtailed appliance ID \\
\hline
\end{tabular}

2) The leader could conduct min-power curtailment, i.e., select appliances from $\mathcal{C}_{t}$ so that minimum power usage is curtailed while still meeting $\mathcal{P}_{\max , t}$.

3) With min-appliance curtailment, the leader can curtail the minimum number of appliances from $\mathcal{C}_{t}$ such that power usage can be kept below $\mathcal{P}_{\max , t}$.

Alternatively, if the real-time appliance joined the active set either in or shortly after the power scheduling phase, then it might be practical (again depending on the appliance) to curtail those that most recently joined the active set.

Furthermore, we note that with real-time appliances in the mix, if $\mathcal{C}_{t}=\emptyset$, then the power demand in the home exceeds the target set by the latest pricing information. Thus, all the appliances (new real-time ones and previously active ones) will be supported but the customer will have to pay beyond the target value set for this time interval. This may or may not be problematic, e.g., to meet the target electricity bill amount for the month, as there may be other time intervals in which the customer's demand may fall well below the set target power level. The algorithm proposed here allows a reduction in total demand during peak hours by fully exploiting the presence of schedulable appliances.

Finally, we note that in some practical scenarios, our proposed admission control and curtailment control mechanism can be easily extended to accommodate schedulable appliances with deadlines. By employing the P_deadline field of the power request message, the priority of schedulable appliances would be changed to real-time appliances when approaching the deadlines. In this case, a modified admission control policy would be needed that accounts for the deadline parameter in scheduling the schedulable appliances that join the active set. Due to space limits, we do not explore these scenarios in the results presented below.

\section{Simulation Results}

\section{A. Simulation Model}

In the simulations, we assume

- The three phases within a scheduling frame have duration $T_{1}=4.5 \mathrm{~min}, T_{2}=29.999 \mathrm{~s}$ and $T_{3}=1 \mathrm{~ms}$, i.e., the frame duration is $T_{f}=T_{1}+T_{2}+T_{3}=5 \mathrm{~min}$;

- The beacon signal and each packet are $1 \mathrm{~ms}$ long and thus the number of slots in the power request phase is $w=298$ (not including the beacon signal); and

- Each appliance is one of $L$ types, where each type has its own power requirement level and mean duration of use. We assume here that power level $\mathcal{P}_{i}=k \varepsilon, i=$ $1, \cdots, N, k=1, \cdots, L$, which is equally distributed, i.e., $p_{k} \stackrel{\text { def }}{=} \operatorname{Pr}\left(\mathcal{P}_{i}=k \varepsilon\right)=1 / L$. In the simulations, we choose $L=5$ and $\varepsilon=100 \mathrm{~W}$. Further, for simplicity, we assume all $L$ appliance types have a duration of use that is exponentially distributed with mean $1 / \mu$. 


\section{B. Comparing Usage With and Without Scheduling}

In Fig. 6, we show one example setting of price profile, minimum and maximum power consumption levels and use them to determine the optimal total power budget $\mathcal{P}_{\max , t}$ according to (4). The price profile is based on the daily report from New York independent system operator (NYISO) [9]. From the plot, we observe that the optimal $\mathcal{P}_{\max , t}$ intends to assign more power budget to the time slots with lower price.
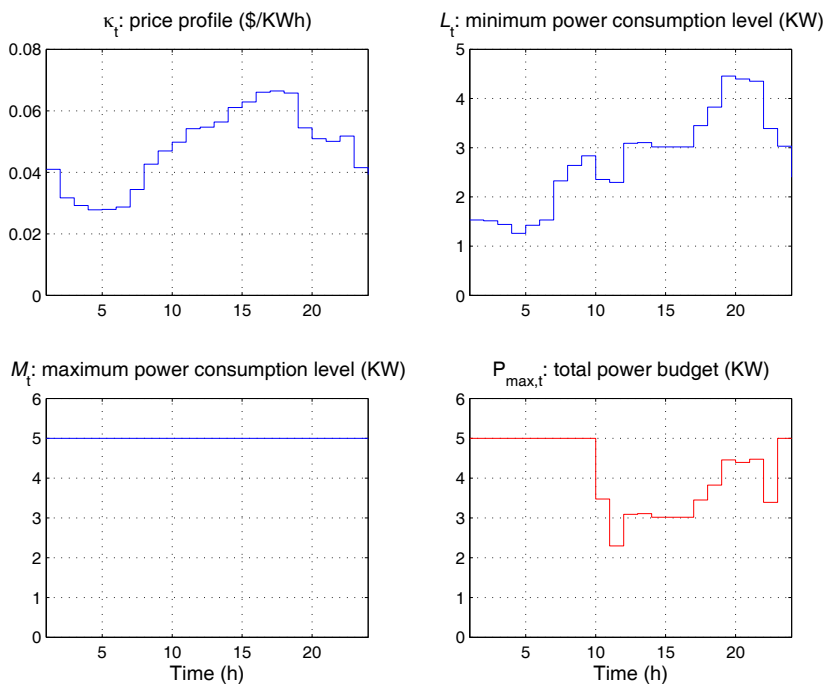

Fig. 6. Optimal total power budget $\mathcal{P}_{\max , t}$. In the simulations, we assume $\mathcal{E}_{0}=100 \mathrm{~kW}$.

Fig. 7 shows the total power consumption with and without power scheduling using the total power budget shown in Fig. 6. In this simulation, we varied the mean arrival rate of appliances based on time-of-day. Specifically, we assume an arrival rate of $\lambda=1 / 1800$ during hours $[1: 7] ; \lambda=1 / 600$ during hours [ $8: 17]$ and $[23: 24]$; and $\lambda=1 / 200$ during hours $[18: 22]$. We see that without power scheduling, the total power consumption can reach up to $9 \mathrm{~kW}$ whereas our proposed scheme keeps total power consumption below the target value $\mathcal{P}_{\text {max }, t}$. We next focus on the delay implications of time-shifting schedulable devices.

\section{Delay Characteristic With Power Scheduling Protocol}

To better understand the delay characteristic of our proposed power scheduling protocol, we assume $\mathcal{P}_{\text {max }, t}=\mathcal{P}_{\max }$ and a fixed arrival rate of $\lambda$ for all hours. Let us define $\eta$ as the probability that total unscheduled power consumption exceeds $\mathcal{P}_{\max }$. We see that $\eta$ can be approximated as $\mathbb{E}\left\{T_{e}\right\} / T_{t}$, where $T_{e}$ is the time duration that total power consumption exceeds $\mathcal{P}_{\max }$ and $T_{t}$ is the total time duration. First we plot the average delay with power scheduling as a function of $\eta$ in Fig. 8. We see that when $\eta$ increases, the average delay increases accordingly. It is worth mentioning that when $\eta=0$ (i.e., when the total power consumption without scheduling will never exceed $\mathcal{P}_{\max }$ ), there is still a delay in the system with power scheduling; it is the overhead of power scheduling protocol and is approximately equal to $T_{f} / 2+T_{2}+T_{3}=180 \mathrm{~s}$.

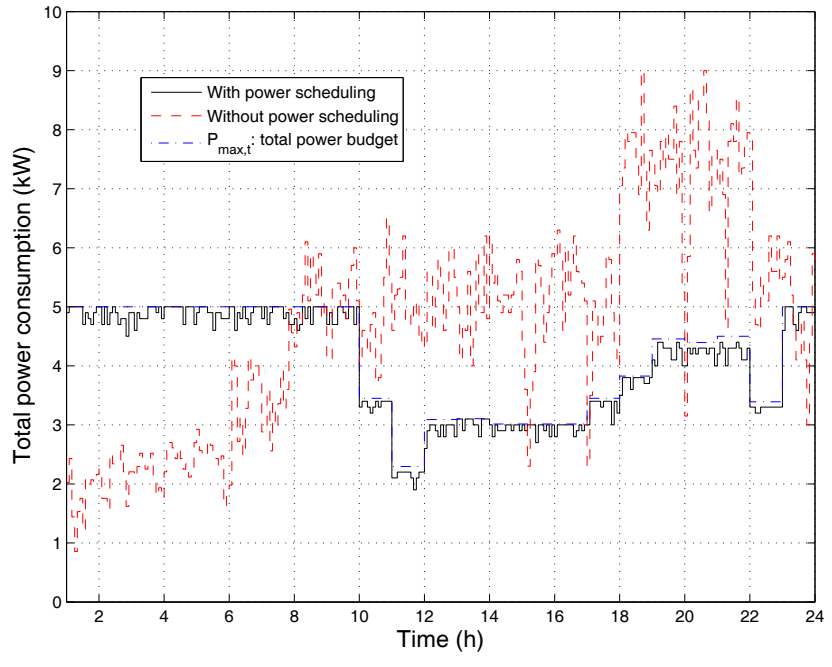

Fig. 7. Total power consumption with and without power scheduling. In the simulations, we choose $N=30, \mu=1 / 3600$ and random ACM.

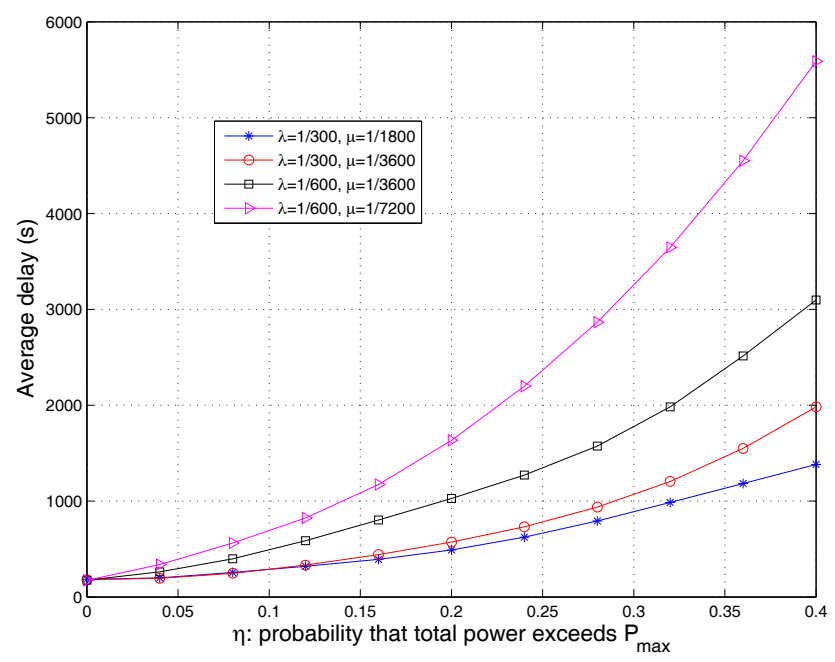

Fig. 8. Average delay versus $\eta$ with various $(\lambda, \mu)$ s. In the simulations, we choose random ACM and $N=15$.

In Fig. 9, we plot the average delay as a function of $\mathcal{P}_{\max }$ with different ACMs. As expected, we see that the average delay decreases as $\mathcal{P}_{\max }$ increases. We also note that the average delays of the different ACMs are very close, especially for large $\mathcal{P}_{\max }$. Since random ACM has linear implementation complexity, it may be a desirable option in these cases.

\section{Delay Characteristic With Real-Time Appliances}

Fig. 10 shows the average delay versus target power budget $\mathcal{P}_{\max }$ with different number real-time appliances in the system. We present results for the random CCM policy only as the performance of the others matches closely. We see that with more real-time appliances in the system, the average delay for scheduable appliances will become larger. This is primarily due to the fact that when one scheduable appliance is curtailed by the leader appliance, it needs to re-compete with other appliances to join the active set. It is also interesting to note that the average delay for all appliances is almost same 


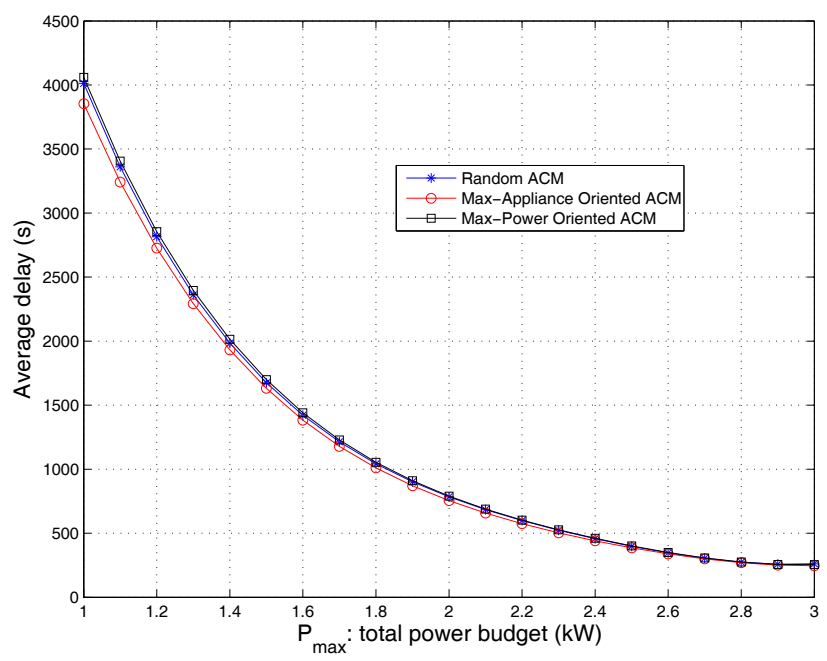

Fig. 9. Average delay versus $\mathcal{P}_{\max }$ with various ACMs. In the simulations, we choose $N=15, \lambda=1 / 300$ and $\mu=1 / 3600$.

with different number of real-time appliances. This indicates that the occurrence of real-time appliances will not increase average delay in the system.

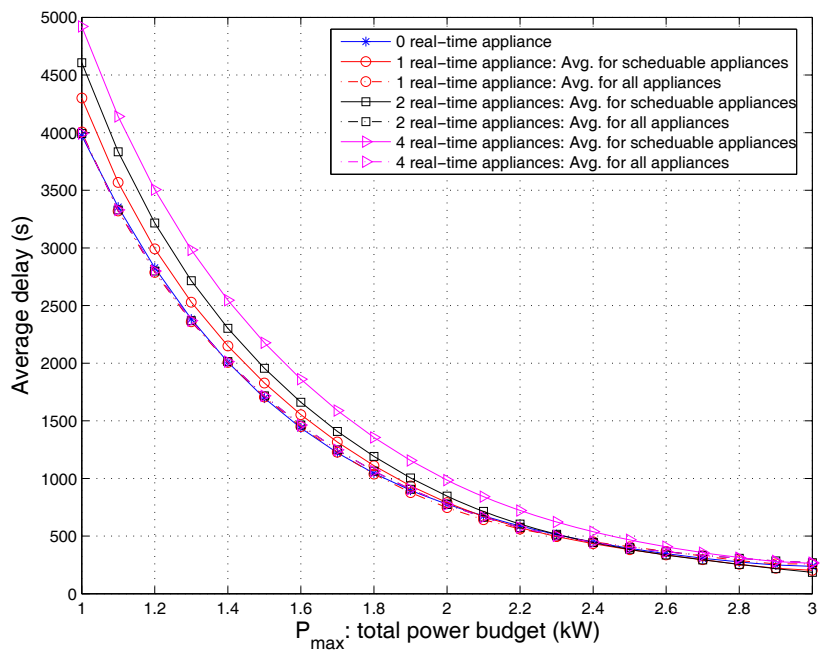

Fig. 10. Average delay versus $\mathcal{P}_{\max }$ with various number of real-time appliances. In the simulations, we choose $N=15, \lambda=1 / 300$ and $\mu=$ $1 / 3600$.

In Fig. 11, we plot the average number of curtailed appliances versus $\mathcal{P}_{\max }$ with different number of real-time appliances. As expected, we see that with larger number of real-time appliances or smaller $\mathcal{P}_{\max }$, the average number of curtailed appliances becomes larger.

\section{CONCLUSION}

In this paper, we propose a power scheduling protocol for demand response in smart grid system. In particular, we develop a joint media access and appliance scheduling approach to enable appliances to coordinate power usage so that total power demand is kept below $\mathcal{P}_{\text {max }, t}$. Simulation results indicate that for an appropriate $\mathcal{P}_{\text {max }, t}$, our proposed scheme leads to a reduced peak demand for the home. In this

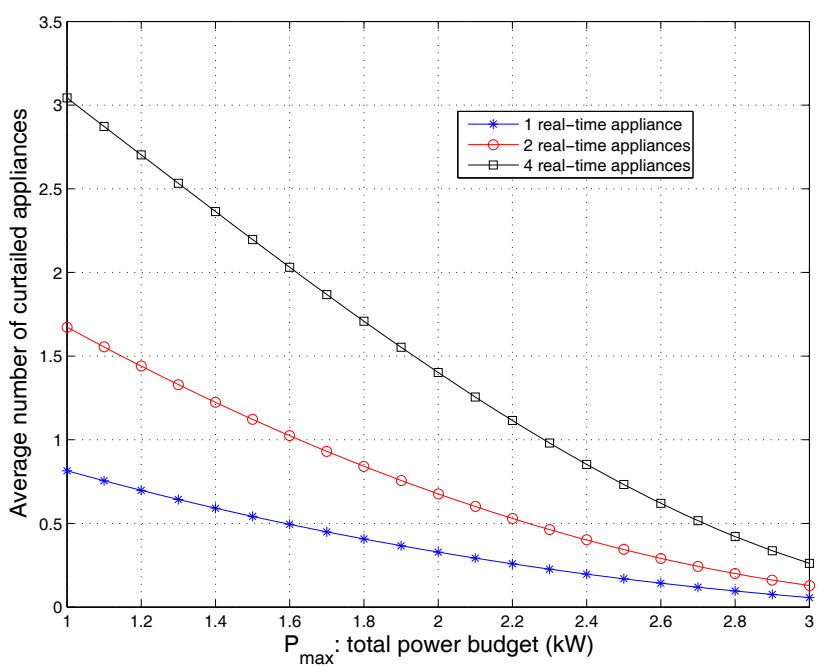

Fig. 11. Average number of curtailed appliances versus $\mathcal{P}_{\max }$ with various number of real-time appliances. In the simulations, we choose random CCM, $N=15, \lambda=1 / 300$ and $\mu=1 / 3600$.

paper, we note that in some scenarios when not enough power budget can accommodate real-time appliances (e.g. a few more real-time appliances use the power simultaneously), the power consumption level will exceed the power budget threshold and the customers will in turn pay more. In the future, we will investigate power scheduling protocols in more practical scenarios.

\section{REFERENCES}

[1] K. Wacks. GridWise Architecture Council/NIST Home-to-Grid Domain Expert Working Group, April 2009. Online available: http://collaborate. nist.gov/twiki-sggrid/pub/SmartGrid/H2G/H2G-Req5.pdf.

[2] Pennsylvania Act 129, 2008. Online available: http://www.puc.state.pa us/electric/Act_129_info.aspx.

[3] D. L. Ha, M. H. Le, and S. Ploix. An Approach for Home Load Energy Management Problem in Uncertain Context. Proc. of IEEE International Conference on Industrial Engineering and Engineering Management, pages 336-339, 2008.

[4] K. S. Choi, Y. K. Ahn, Y. C. Park, W. C. Park, H. M. Seo, K. M. Jung, and K. H. Seo. Architectural Design of Home Energy Saving System Based on Realtime Energy-Awareness. Proc. of 4th International Conference on Ubiquitous Information Technologies and Applications, pages 1-5, 2009.

[5] S. Tompros, N. Mouratidis, M. Draaijer, A. Foglar, and H. Hrasnica. Enabling Applicability of Energy Saving Applications on the Appliances of the Home Environment. IEEE Netw., 23(6):8-16, 2009.

[6] Y. S. Son and K. D. Moon. Home Energy Management System Based on Power Line Communication. Proc. of IEEE International Conference on Consumer Electronics, pages 115-116, 2010.

[7] M. Inoue, T. Higuma, Y. Ito, N. Kushiro, and H. Kubota. Network Architecture for Home Energy Management System. IEEE Trans. Consum. Electron., 49(3):606-613, 2003.

[8] H. Kellerer, U. Pferschy, and D. Pisinger. Knapsack Problems. Berlin: Springer, 2004.

[9] Federal Energy Regulatory Commission. New York Independent System Operator (NYISO) Daily Report. 2010. Available Online: http://www. ferc.gov/market-oversight/mkt-electric/new-york/nyiso-archives.asp. 


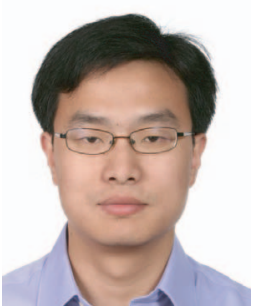

WiMAX systems.

His current research interests include PHY and MAC layer wireless communication, cross-layer design and optimization of wireless networks.

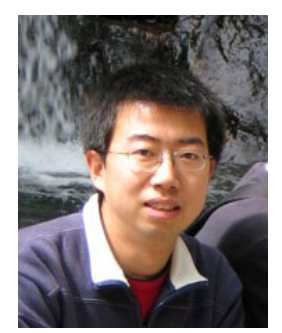

Chen Chen received his M.Sc. and B.Sc degrees from Xi'an Jiaotong University, Xi' an, China in 2006 and 2009 respectively, both in electrical engineering. He is currently working towards his Ph.D. degree in electrical and computer engineering at Lehigh University, Bethlehem, PA.

His current research interests include applications of game theory and communications \& control theory in smart grid.

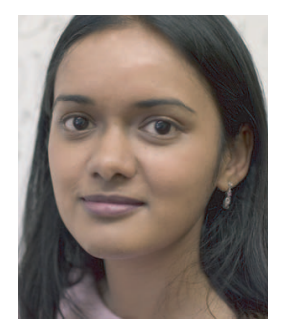

Shalinee Kishore is an Associate Professor in the Department of Electrical and Computer Engineering at Lehigh University in Bethlehem, Pennsylvania. She obtained the Ph.D. and M.A. degrees in Electrical Engineering from Princeton University in 2003 and 2001, respectively, and the M.S. and B.S. degrees in Electrical Engineering from Rutgers University in 1999 and 1996, respectively. From 1994 to 2002, she has held numerous internships at AT\&T, Bell Labs, and AT\&T Labs-Research. Prof. Kishore is the recipient of the Presidential Early Career Award for Scientists and Engineers, the National Science Foundation CAREER Award, the P.C. Rossin Assistant Professorship, and the AT\&T Labs Fellowship Award. She has also served as a Kavli Fellow for the National Academy of the Sciences.

Her research interests are in communications theory, networks, and signal processing, with emphasis on wireless systems.
Aylin Yener received two B.Sc. degrees, with honors, in electrical and electronics engineering, and in physics, from Boğaziçi University, Istanbul, Turkey, in 1991, and the M.S. and Ph.D. degrees in electrical and computer engineering from Rutgers University, New Brunswick, NJ, in 1994 and 2000, respectively.

During her Ph.D. studies, she was with the Wireless Information Network Laboratory (WINLAB), Department of Electrical and Computer Engineering, Rutgers University. From September 2000 to December 2001, she was with the Electrical Engineering and Computer Science Department, Lehigh University, PA, where she was a P.C. Rossin Assistant Professor. In January 2002, she joined the faculty of The Pennsylvania State University, University Park, where she was an Assistant Professor, then Associate Professor, and is currently Professor of Electrical Engineering. During the academic year 20082009, she was a Visiting Associate Professor with the Department of Electrical Engineering, Stanford University, Stanford CA. Her research interests are in information theory, communication theory and network science, with emphasis on fundamental limits of wireless ad hoc networks and information theoretic security.

Dr. Yener received the NSF CAREER award in 2003 and is a member of the team that received the DARPA Information Theory for Mobile Ad Hoc Networks (ITMANET) Young Investigator Team Award in 2006. In 2010, she received the Penn State Engineering Alumni Society (PSEAS) Outstanding Research Award, and the Best Paper award from the Communication Theory Symposium in IEEE International Conference on Communications (ICC). Her service to IEEE includes membership in the Technical Program Committees of various annual conferences since 2002. She chaired the Communications Track in the Asilomar Conference on Signals, Systems and Computers in 2005 and in 2008. She served as the Technical Program Co-Chair for the Communication Theory Symposium of the IEEE International Conference on Communications (ICC) 2009 and for the Wireless Communications Symposium of the IEEE International Conference on Communications (ICC) 2008. She currently serves as an editor for the IEEE TRANSACTIONS ON WIRELESS COMMUNICATIONS and for the IEEE TRANSACTIONS ON COMMUNICATIONS. Her service to the IEEE Information Theory Society includes chairing the Student Committee since September 2007. She is the cofounder of the Annual School of Information Theory in North America, and served/serves as the general Co-Chair of the First Annual School of Information Theory that was held at Penn State University, University Park, in June 2008, the Second Annual School of Information Theory at Northwestern University, Evanston, IL, in August 2009, and the Third Annual School of Information Theory at University of Southern California, Los Angeles, in August 2010. 